\title{
Effects of Food-Specific IgG on Health Outcomes of Asymptomatic Physical Examination Population
}

\section{Mingxia Wu}

Third Military Medical University Southwest Hospital

\section{Xiaofang Wang}

Third Military Medical University Southwest Hospital

\section{Li Sun}

Third Military Medical University Southwest Hospital

\section{Zongtao Chen ( $\nabla$ zongtaochen@126.com )}

Third Military Medical University Southwest Hospital

\section{Research}

Keywords: Food-specific IgG, Physical Examination population, Health Outcomes, Body weight, Ttriglyceride, Fasting blood glucos

Posted Date: October 12th, 2021

DOI: https://doi.org/10.21203/rs.3.rs-958853/v1

License: (1) (1) This work is licensed under a Creative Commons Attribution 4.0 International License. Read Full License 


\title{
Effects of Food-Specific IgG on Health Outcomes of Asymptomatic Physical Examination Population
}

\author{
Mingxia Wu,Xiaofang Wang,Li Sun,Zongtao Chen*
}

Health Management Center, First Affiliated Hospital of Army Medical University (Third Military Medical University), Chongqing 400038, China *corresponding authors. E-mail address: zongtaochen@126.com 


\begin{abstract}
:
Background:Although food-specific $\operatorname{IgG}$ is associated with the development and progression of some diseases as shown by many studies, it is also present in the population without clinical symptoms. However, the damage of the immune response it evokes to the health of such population has not been studied yet. Methods: The asymptomatic physical examination population (APEP) was selected according to the inclusion and exclusion criteria, and the physical examination data were collected. The subjects were divided into IgG-positive group (IgG-po group) and IgG-negative group (IgG-neg group). The hematologic and imaging examination results were compared between the two groups, and their odds ratios (OR) and 95\% confidence interval $(95 \% \mathrm{CI})$ were calculated using binary logistic regression to determine the relationship between food-specific IgG and different health outcomes. Results: The data of 28,292 subjects were included in the analysis of the study. The overall IgG positive rate was up to $52.3 \%$, with mild to moderate IgG positive predominantly. Compared with IgG negative group, IgG positive group was associated with a decrease in the risks of hypertriglyceridemia, abnormal fasting blood glucose and overweight $(\mathrm{OR}=0.87,95 \%$ :CI:0.83-0.92; OR=0.93,95\%CI:0.87-0.99; OR=0.92, 95\% CI:0.87-0.96) and with an increase in the incidence rate of thyroid nodule $(\mathrm{OR}=1.09,95 \% \mathrm{CI}: 1.04-1.15)$. Conclusion:Food-specific $\mathrm{IgG}$ is generally present in APEP and has an unique distribution profile, which plays a positive role in maintaining normal body weight and metabolic indicators(triglyceride(TG) and fasting blood glucose(FBG)), without influencing the nutritional status. The physiological mechanism is worth further study.
\end{abstract}

Key words:Food-specific IgG, Physical Examination population, Health Outcomes,Body weight,Ttriglyceride,Fasting blood glucose 


\section{Introduction}

At present, the role of food-specific IgG in the health of human body remains controversial. Numerous studies have suggested that food-specific IgG is involved in the development and progression of some diseases, such as inflammatory bowel disease[1-4], irritable bowel syndrome[5], migraine[6, 7] and mental disease [8-11], of which the symptoms can be relieved by food-specific IgG-based diet recommendations [12-17]. IgG antibody can form an immune complex with allergens in foods and thus induce the body's mild inflammatory reactions[18] that are manifested as various system symptoms and diseases. According to another stud, however, it provides natural protection against food allergy[19, 20]. The most direct evidence comes from an oral immunotherapy (OIT) study showing that OIT for peanuts can remarkably induce an increase in the plasma level of food-specific IgG [20-22], which indicates the involvement of $\operatorname{IgG}$ in mitigating the symptoms and inducing food tolerance of allergic patients.

Previous clinical studies on food-specific IgG typically focused on the ill population, with the asymptomatic population as control group; some study demonstrated the presence of food-specific IgG in the healthy people [9], but this important finding is generally ignored. If food-specific IgG is associated with the development and progression of diseases, will such permanent IgG positive state affect the health of asymptomatic population? To answer this question, this study was designed to analyze the test results of food-specific IgG and the physical examination conclusions of the large-sized asymptomatic physical examination population (APEP).

\section{Methods}

\subsection{Study design and participants}

The study data came from APEP who firstly received the test of 14 food-specific IgGs in Health Management Department of the First Affiliated Hospital of Army Medical University during 2010-2020 and met the following criteria: age $\geq 18$ years, no previous food intolerance test, no food intake restriction, and no food allergic symptoms or resultantly medical care requirement. The data without such information as age, sex, height and body weight were excluded. Other physical examination data were also collected, including blood pressure, waist circumference, hematologic examination indicators (e.g., total cholesterol (TC), TG, low density lipoprotein cholesterol (LDL-C), high-density lipoprotein cholesterol (HDL-C), FBG, uric acid(UA), plasma-albumin(ALB), liver function, and blood routine (dominantly hemoglobin), and other examination items (e.g., thyroid ultrasonography, pulmonary radiography, abdominal ultrasonography, bone densitometry, gastroscopy, enteroscopy, and prostatic ultrasonography for men, and breast ultrasonography, uterus \& appendages ultrasonography, and cervical cancer screening for women).

After screening, a total of 28292 physical examination subjects were included in the statistical analysis (32876 subjects were preliminarily screened, of whom 3744 and 840 were excluded due to a lack information of sex, height and body weight and 
for this was not the first food-specific antibody test, respectively), and the various examinations they completed are shown in Figure 1. All subjects were divided into IgG-po group and IgG-neg group, and the above examination and test results in two groups were statistically analyzed (Table 1). To further investigate the relations of food-specific IgG with the statistically different health outcomes, we established a model with the available data. Specifically, we combined the influential factors of health outcomes reported previously, included factors such as age, sex, food-specific IgG, body weight, blood pressure, blood lipid, blood glucose, uric acidUA and fatty liver, and then calculated their OR and 95\%CI using binary logistic regression. This study was approved by the Ethics Committee of the First Affiliated Hospital of Army Medical University, and as all data were retrospectively analyzed in an anonymous form, the written informed consent from subjects was waived.

\subsection{Definition of positive}

In the present study, food-specific IgG test was performed by detecting the serum IgG level with enzyme-linked immunosorbent assay (ELISA) and using 14 foods most frequently eaten by Chinese people (e.g., pork, rice, chicken, shrimp, corn, milk, soybean, wheat, mushroom, beef, cod, crab, egg and tomato). Based on different food-specific IgG levels, there were four grades: $<50 \times 10^{3} \mathrm{U} / \mathrm{L}$, negative (NEG); $50-100 \times 10^{3} \mathrm{U} / \mathrm{L}$, mild positive (MIP); $100-200 \times 10^{3} \mathrm{U} / \mathrm{L}$, moderate positive (MOP); and $>200 \times 10^{3} \mathrm{U} / \mathrm{L}$, severe positive (SEP). Based on the number of IgG positive foods, IgGs can be classified into Single food-specific positive (SIPO) and multiple food-specific positive (MUPO).

Based on body mass index $(\mathrm{BMI})\left(\mathrm{BMI}=\right.$ body weight $/ \mathrm{height}^{2}\left(\mathrm{Kg} / \mathrm{m}^{2}\right)$, people were classified into three types: underweight $\left(\mathrm{BMI}<18.5 \mathrm{Kg} / \mathrm{m}^{2}\right)$, normal $\left(18.5 \mathrm{Kg} / \mathrm{m}^{2} \leq \mathrm{BMI}<24.0 \mathrm{Kg} / \mathrm{m}^{2}\right)$ and overweight $\left(\mathrm{BMI} \geq 24.0 \mathrm{Kg} / \mathrm{m}^{2}\right)$. The relevant definitions were as follows: adult central obesity, waist circumference $\geq 90 \mathrm{~cm}$ (for men) and $\geq 85 \mathrm{~cm}$ (for women); abnormal blood pressure, systolic blood pressure (SBP) $\geq 140 \mathrm{mmHg}$ or diastolic blood pressure (DBP) $\geq 90 \mathrm{mmHg}$; abnormal blood lipid, anyone of $\mathrm{TG}>1.73 \mathrm{mmol} / \mathrm{L}, \quad \mathrm{TC}>5.7 \mathrm{mmol} / \mathrm{L}, \quad \mathrm{LDL}-\mathrm{C}>3.1 \mathrm{mmol} / \mathrm{L} \quad$ and HDL-C $<0.9 \mathrm{mmol} / \mathrm{L}$; abnormal $\mathrm{FBG}, \quad \mathrm{FBG} \geq 6.1 \mathrm{mmol} / \mathrm{L} ; \quad$ hyperuricemia, $\mathrm{UA}>428 \mathrm{umol} / \mathrm{L}$; abnormal liver function, alanine aminotransferase or aspartate aminotransferase $>42 \mathrm{IU} / \mathrm{L}$; decreased ALB: ALB $<38 \mathrm{~g} / \mathrm{L}$; anemia: hemoglobin $(\mathrm{HB})<$ $120 \mathrm{~g} / \mathrm{L}$ (for men) and $<110 \mathrm{~g} / \mathrm{L}$ (for women); abnormal renal function, blood creatinine $(\mathrm{Cr})>104 \mathrm{umol} / \mathrm{L}$; abnormal bone mineral density, including osteopenia $(-2.5<\mathrm{T}<-1.0)$ and osteoporosis $(\leq-2.5)$; lung lesions, including pulmonary nodule, emphysema, lung cyst, lung cancer; gastrointestinal lesions, including gastrointestinal inflammation, polyp, ulcer, and cancer; breast lesions, including breast nodule, breast cyst, mammary duct lesions, and breast cancer; abnormal gynecological ultrasonography, including uterine and ovarian nodular lesions or canceration; abnormal cervical liquid-based cytological test, including low-grade and high-grade squamous intraepithelial lesions, and cervical cancer.

\subsection{Statistical analysis}

The quantitative data of normal distribution were presented as mean \pm standard deviation $(\mathrm{x} \pm \mathrm{S})$ and analyzed for pair-wise comparison and multiple comparisons 
with independent sample $t$ test and analysis of variance (ANOVA), and those of skewed distribution were expressed as median and analyzed with Wilcoxon and Kruskal-Wallis rank sum tests. The qualitative data were analyzed with $\chi^{2}$ test. The OR and $95 \% \mathrm{CI}$ were calculated using binary logistic regression to determine the relationship between food-specific IgG and different healthy outcomes. All statistical analyses in this study were conducted using SPSS 23.0 software. A P-value $<0.05$ was considered statistically significant.

\section{Results}

\subsection{Distribution profile of food-specific IgG in APEP}

The overall IgG positive rate was up to $52.3 \%$. The top seven IgG-positive foods were egg (IgG positive rate: $29.0 \%)$, crab $(10.89 \%)$, milk $(9.84 \%)$, corn $(8.28 \%)$, tomato $(7.81 \%)$, mushroom $(6.62 \%)$ and shrimp $(6.33 \%)$. The incidence rates of SEP and MUPO were higher in women than in men $\left(12.92 \%\right.$ vs $7.4 \%, \mathrm{x}^{2}=518.61, \mathrm{P}<$ $0.001 ; 29.66 \%$ vs $\left.20.90 \%, x^{2}=464.06, \mathrm{P}<0.001\right)$. Food-specific IgG changed over age, especially with a tendency to increase in crab, mushroom and shrimp (Figure 2).

\subsection{Analysis of health outcomes of IgG group}

Compared with IgG-neg group, IgG-po group had a lower mean age, a higher proportion of women, and lower incidence rates of overweight, central obesity, dyslipidemia, hypertriglyceridemia, hypercholesterolemia and low density lipopreteinemia $(43.80 \pm 11.31$ vs $45.08 \pm 10.39$ years, $\mathrm{t}=9.96, \mathrm{P}<0.001 ; 43.17 \%$ vs $31.49 \%, \mathrm{x}^{2}=409.05, \mathrm{P}<0.001 ; 52.57 \%$ vs $58.37 \%, \mathrm{x}^{2}=113.98, \mathrm{P}<0.001 ; 33.39 \%$ vs $37.61 \%, \mathrm{x}^{2}=49.30, \mathrm{P}<0.001 ; 51.65 \%$ vs $56.79 \%, \mathrm{x}^{2}=74.76, \mathrm{P}<0.001 ; 35.03 \%$ vs $43.27 \%, \mathrm{x}^{2}=201.33, \mathrm{P}<0.001 ; 22.95 \%$ vs $25.80 \%, \mathrm{x}^{2}=31.04, \mathrm{P}<0.001 ; 3.90 \%$ vs $4.71 \%, \mathrm{x}^{2}=9.77, \mathrm{P}<0.002$ ), and the mean blood pressure tended to decrease. However, there was no significant difference in the incidence rate of high density lipopreteinemia between the two groups $\left(21.90 \%\right.$ vs $\left.22.30 \%, \mathrm{x}^{2}=0.64, \mathrm{P}=0.42\right)$. Besides, the incidence rates of abnormal blood glucose $\left(12.59 \%\right.$ vs $15.85 \%, \mathrm{x}^{2}=61.47$, $\mathrm{P}<0.001)$ and abnormal liver function $\left(30.21 \%\right.$ vs $\left.37.11 \%, \mathrm{x}^{2}=152.88, \mathrm{P}<0.001\right)$ and the levels of UA and HB were all lower in IgG-po group, while ALB was not different between two groups $(45.93 \pm 2.76$ vs. $45.91 \pm 2.73 \mathrm{~g} / \mathrm{L}, \mathrm{t}=-0.58, \mathrm{P}=0.56)$.

Abdominal color Doppler ultrasonography showed that the incidence rate of fatty liver, gallbladder polyp and renal calculus was lower in IgG-po group $(31.26 \%$ vs $37.31 \%, \mathrm{x}^{2}=114.75, \mathrm{P}<0.001 ; 8.17 \%$ vs $9.08 \%, \mathrm{x}^{2}=7.50, \mathrm{P}=0.01 ; 3.79 \%$ vs $4.27 \%$, $\mathrm{x}^{2}=4.20, \mathrm{P}=0.06$ ), but that of cholecystolithiasis was not significantly different between IgG-po group and IgG-neg group. Compared with IgG-neg group, the incidence rate of osteopenia and osteoporosis, as well as male prostatic nodule $(6.81 \%$ vs. $\left.8.40 \%, \mathrm{x}^{2}=5.32, \mathrm{P}=0.02\right)$ was decreased while that of thyroid nodule $(38.99 \%$ vs. $\left.35.82 \%, \mathrm{x}^{2}=21.47, \mathrm{P}<0.001\right)$ was increased in IgG-po group. The results of female breast color Doppler ultrasonography, gynecological ultrasonography and cervical cancer screening showed that there was no significant difference between two groups. (Table 1)

\subsection{Effects of food-specific IgG on various health outcomes}

The above analyses showed that compared with IgG-neg group, the incidence 
rates of overweight, central obesity, abnormal blood lipid (including hypertriglyceridemia, hypercholesterolemia and low density lipopreteinemia), abnormal FBG, abnormal liver function, fatty liver, gallbladder polyp, and renal calculus were decreased while the incidence of thyroid nodule was increased in IgG-po group. Binary logistic regression analysis showed that IgG positive state (vs. IgG negative) was associated with a decrease in the incidence rate of overweight $(\mathrm{OR}=0.92$, 95\%CI: 0.87-0.96) and in FBG (OR=0.93, 95\%CI: 0.87-0.99), and with an increase in the incidence rate of decreased TG (OR=0.87, 95\%CI: $0.83-0.92)$, but did not demonstrate obvious impact on $\mathrm{TC}(\mathrm{OR}=0.95,95 \% \mathrm{CI}$ : 0.90-1.0) and HDL-C (OR=0.98, 95\%CI: 0.88-1.09). In addition, IgG positive state was not significantly associated with abnormal SBP (OR=0.98, 95\%CI: 0.91-1.05) and DPB (OR=0.93, 95\% CI: $0.87-1.0)$, as well as the development of fatty liver $(\mathrm{OR}=0.98,95 \% \mathrm{CI}$ : 0.92-1.04), but IgG positive subjects were more susceptible to thyroid nodule (OR=1.09, 95\%CI: 1.04-1.15). (Table 2)

\subsection{Relations of different degrees and types of food-specific IgG with TG, FBG and BMI}

The above analysis showed that food-specific IgG positive state was correlated with the decreased incidence rates of abnormal FBG, hypertriglyceridemia and overweight. We further investigated the relations of the elevation level of $\operatorname{IgG}$ antibody (MIP, MOP and SEP) and the number of IgG positive foods (SIPO and MUPO) with plasma FBG, TG and BMI. The analysis results suggested that a higher IgG antibody titer responded to lower TG, FBG and $\mathrm{BMI}(\mathrm{H}=372.30, \mathrm{P}<0.001$; $\mathrm{H}=216.67, \mathrm{P}<0.001 ; \mathrm{H}=243.33, \mathrm{P}<0.001$ ), and these three indicators also tended to decrease with an increasing number of $\operatorname{IgG}$ positive foods $(\mathrm{H}=372.30, \mathrm{P}<0.001$; $\mathrm{H}=172.39, \mathrm{P}<0.001 ; \mathrm{H}=312.58, \mathrm{P}<0.001$ ) (Figure 3).

\section{Discussion}

Different from previous studies focusing on the ill population, this study included APEP with no apparent clinical symptoms and no previous dietary guidance for food-specific IgG test results. By analyzing food-specific IgGs and physical examination results of APEP, we found the distribution profile of food-specific IgG in APEP and the possible impact of food-specific IgG-mediated immune response on the health outcomes of APEP.

This study demonstrated that the positive rate of food-specific IgG in the blood of APEP was up to $52.3 \%$, generally with mild to moderate elevation of antibody titer, which was lower than that of ulcerative colitis (57.5\%-70.1\%), Crohn disease $(90.72 \%)[4,16,23]$ and food-specific IgGs associated with clinically allergic symptoms[24]. Such differences indicate that food-specific IgG with a higher positive rate and a greater titer may be associated with some morbid states, but it is not clear whether the disease results in an increase of IgG positive rate or food-specific IgG induces the morbid state. In this study, we investigated 14 foods most frequently eaten by Chinese people and found a significantly higher IgG positive rate in women than in men, which perhaps is somewhat associated with a greater incidence rate of autoimmune disease (AI) in women than in men, as shown by the previous studies[25, 
26]. The positive rate of most food-specific IgG antibodies decreased over age in the follow-up, but it was not always the case. The above positive rate in some foods (e.g., crab, mushroom and shrimp) tended to increase over age, while a previous study found a negative correlation of $\mathrm{IgG}$ level in all foods with age[27].Another study showed that in Northeast China, a higher food-specific IgG positive rate was observed in crab, shrimp, egg white/yolk, milk and fish[28], which was greatly different from our study finding that the food-specific IgG positive rate was higher in egg, crab, milk, corn and tomato. It suggests that the food habits in different areas may influence the type of food-specific IgG, which was not observed in our study because we adopted a large sample size from the same area.

It is believed that food-specific IgG is involved in the body's complex immune response[28]. Some foods cannot be completely digested by human body due to a lack of corresponding enzymes, and they are recognized as foreign substances in gastrointestinal mucosal lymphatic tissues and then form immune complexes with specific IgG antibodies in the body; thereafter, macromolecular complexes are phagocytized by monocytes, while micromolecular complexes are excreted by kidneys. It remains unknown whether such IgG-mediated immune response has some common effects on the physical examination results of APEP. This study showed no difference in the incidence rate of abnormal renal function between the two groups. In IgG-neg group, the incidence rate of renal disfunction tended to decrease and that of fatty liver was much lower, but after the adjustment of influential factors reported in the literature, IgG positive state demonstrated no apparent impact, which indicates that IgG has no significant effect on liver and renal functions. Besides, there were significant differences in the incidence rates of health outcomes (including abnormal blood pressure, hyperuricemia, gallbladder polyp, renal calculus, and prostatic nodule) between two groups (Table 1), but IgG positive state had no statistically significant influence on these health outcomes after adjusting age, sex and other reported influential factors.

Previous studies have revealed that food-specific IgG may affect the body's nutritional status[16], but no difference in ALB was found between the two groups in this study. Although the incidence rate of anemia was higher in IgG-po group, food-specific IgG exhibited no effect of aggravating anemia after excluding factors such as age and sex. Food-specific IgG positive state was related with a decreased risk of hypertriglyceridemia, abnormal FBG and overweight, suggesting that it may be an independent protecting factor of the above indicators and work better with a higher titer and in a larger number of foods (Figure 3). Such phenomenon was firstly found in APEP, which is similar to the results of an in vivo animal experiment by N. V. Batista et al[29]. They found a decrease in body weight, blood glucose and blood lipid, which was followed by a decline of plasma IgE level, a gradual elevation of IgG level, and a slight decrease of metabolic indicators (as compared with at the allergic state) in the mice treated with sensibiligens after continuing the food antigen stimulation. These similar metabolism characteristics suggest that food-specific IgG is somewhat associated with food allergy.

As we know, acute food allergy and chronic food allergy are mainly related with 
some co-factors[30]. In fact, the diagnosis of food allergy is a clinical challenge and requires history inquiry and food exclusion in most cases[31, 32], and it is very difficult to make in patients without apparent symptoms. In our study, IgG positive subjects might be in a non-obvious food allergy state initially after contacting some foods and no restrictions are imposed on food intake in daily life due to an absence of significant symptoms. Similarly, there was a long-time repeated food antigen stimulation which induced the tolerance of immune system; thus food-specific IgG with corresponding metabolic changes was detected in the body of these subjects. It is just coincident to another view of food-specific IgG: IgG confers natural prevention against food allergy[33]. IgG can send negative signals after binding with FcgRIIb receptor in mast cells, and can also serve as a spatial blockage antibody to interrupt the binding of $\operatorname{IgE}$ and mast cells and thus suppress allergy [19]. The mentioned effects of IgG have been confirmed in the clinical studies. For example, Savilahti EM et al found that the tolerance of milk allergy in children was associated with the blood IgG level [34], In addition, maternal food-specific IgG may be correlated with the food tolerance of offspring [35].

\section{5.conclusion}

In conclusion, food-specific IgG is generally present in APEP, predominantly with mild to moderate elevation. Such distribution feature exhibits no significant tendency of inducing diseases except increasing the risk of thyroid nodule. However, it cannot be excluded that if $\operatorname{IgG}$ antibodies have a higher positive rate and a greater titer, foods, as an important environmental factor, may be involved in the development and progression of some diseases. For populations with a lower BMI, dietary guidance can be provided according to IgG test results, because IgG positive state may further decrease BMI. For patients with overweight and obesity, food-specific IgG plays a positive role in maintaining normal body weight and metabolic indicators (mainly TG and FBG), without influencing the nutritional status. The physiological mechanism is worth further study.

\footnotetext{
Abbreviations

APEP,asymptomatic physical examination population;FBG,fasting blood glucose; BMI,body mass index ;BP,blood pressure;SBP,Systolic blood pressure;DBP,Diastolic blood pressure; LDL-C,low density lipoprotein cholesterol ;HDL-C,high density lipoprotein cholesterol ;TC,total cholesterol ;TG,triglyceride;UA, uric acid;ALB, plasma albumin;HB,hemoglobin;Cr,creatinine; $\mathrm{CI}$, confidence interval; OR, odds ratio;MIP:Mild positive;MOP:Moderate positive;SEP:Severe positive;SIPO:A food-specific IgG positive;MUPO:Multiple food-specific IgG positive.
}

\section{Acknowledgements}

Not applicable

\section{Authors' contributions}


All authors participated in this work with their substantive contributions. MXW and ZTC carried out the manuscript preparation and statistical analysis.MXW was a major contributor in writing the manuscript. MXW,XFW and LS had the responsibility of data collection. ZTC had participated in the review of the paper. All authors read and approved the final manuscript.

\section{Availability of data and materials}

The datasets used and/or analysed during the current study are available from the corresponding author on reasonable request.

\section{Ethics approval and consent to participate}

The study was conducted according to the Declaration of Helsinki. This study was approved by the Ethics Committee of the First Affiliated Hospital of Army Medical University, and as all data were retrospectively analyzed in an anonymous form, the written informed consent from subjects was waived.

\section{Consent for publication}

Not applicable.

\section{Competing interests}

The authors declare that they have no competing interests.

\section{Funding}

Not applicable. 


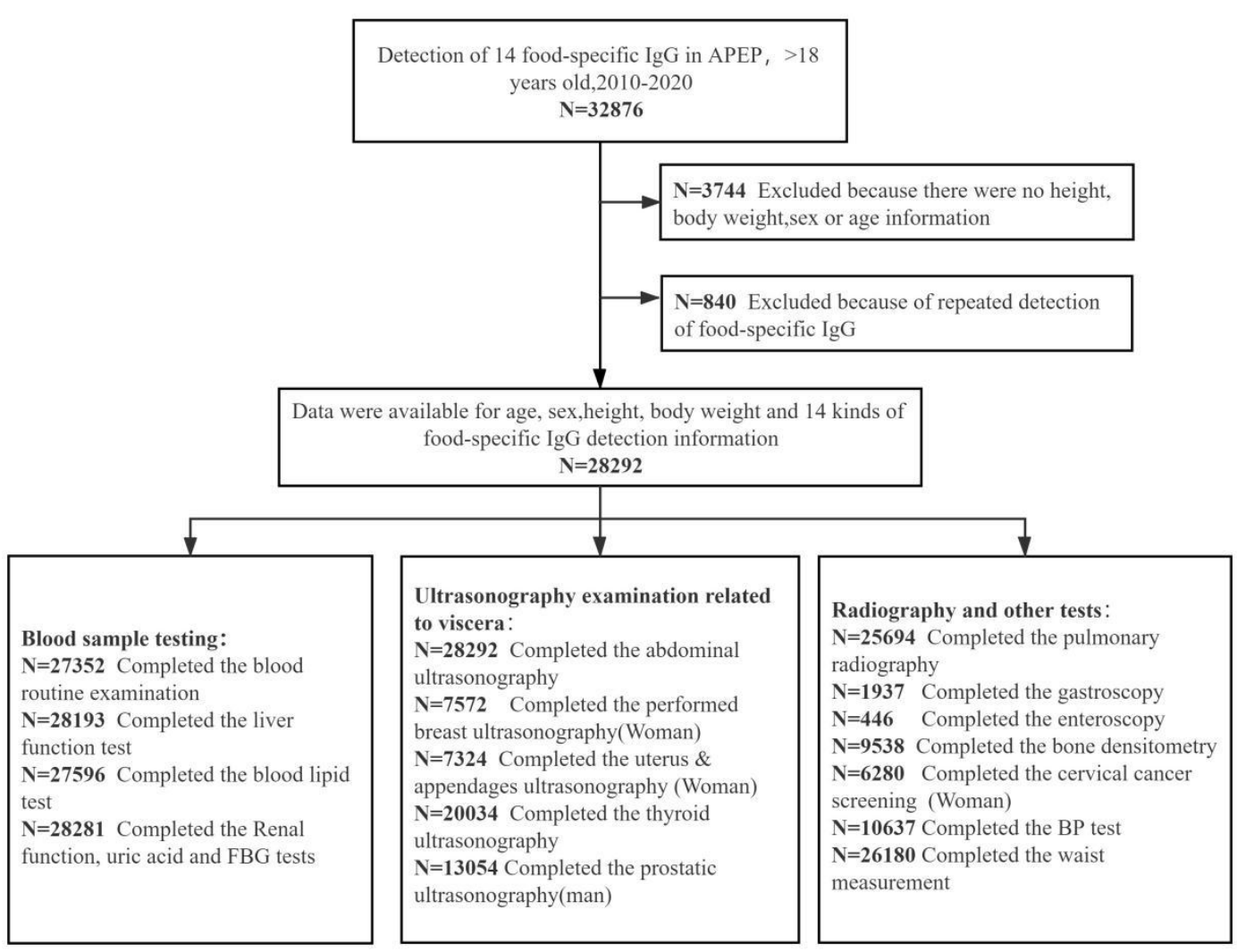

Figure 1. Flow chart of study population .(APEP,asymptomatic physical examination population; FBG,fasting blood-glucose.BP,blood pressure.) 

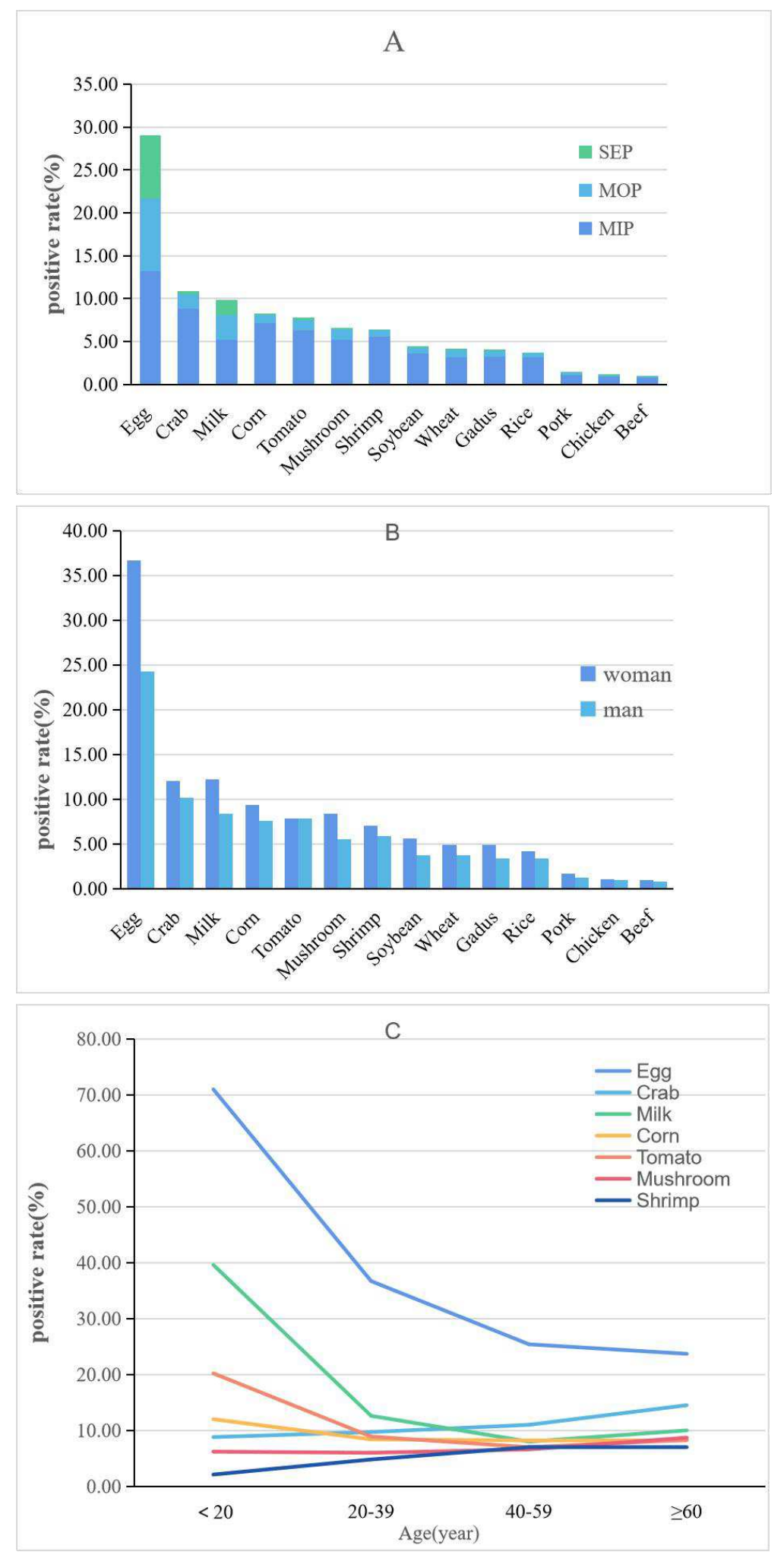

Figure 2.Distribution of food-specific IgG in the APEP.(A:The positive rate of 14 kinds of common food.MIP:Mild positive,MOP:Moderate positive,SEP:Severe positive;B:The positive status of food-specific IgG in different sex; $\mathbf{C}$ :The trend of food-specific IgG positive rate with age (seven foods with higher positive rate)). 
Table 1. Conclusions of various physical examinations grouped according to food-specific IgG

\begin{tabular}{|c|c|c|c|c|}
\hline & IgG-neg group & IgG-po group & $t / x^{2}$ & $\mathrm{P}$ \\
\hline Age (year) & $45.08 \pm 10.39$ & $43.8 \pm 11.31$ & 9.96 & $<0.001$ \\
\hline \multicolumn{5}{|l|}{ Gender } \\
\hline Woman(n/\%) & $4170 / 31.49$ & $6497 / 43.17$ & 409.05 & $<0.001$ \\
\hline $\operatorname{Man}(\mathrm{n} / \%)$ & $9072 / 68.51$ & $8553 / 56.83$ & & \\
\hline \multicolumn{5}{|l|}{ Distribution of BMI } \\
\hline$<18.5 \mathrm{Kg} / \mathrm{m}^{2}(\mathrm{n} / \%)$ & $284 / 2.14$ & $498 / 3.31$ & 113.98 & $<0.001$ \\
\hline $18.5-23.9 \mathrm{Kg} / \mathrm{m}^{2}(\mathrm{n} / \%)$ & $5228 / 39.48$ & $6640 / 44.12$ & & \\
\hline$\geq 24.0 \mathrm{Kg} / \mathrm{m}^{2}(\mathrm{n} / \%)$ & $7730 / 58.38$ & $7912 / 52.57$ & & \\
\hline Central obesity (n/\%) & $4589 / 37.61$ & $4668 / 33.39$ & 49.3 & $<0.001$ \\
\hline Dysarteriotony & $1242 / 22.20$ & $984 / 19.51$ & 11.13 & 0.001 \\
\hline $\mathrm{SBP}(\mathrm{mmHg})$ & $122.89 \pm 18.12$ & $121.66 \pm 17.72$ & 3.52 & $<0.001$ \\
\hline $\mathrm{DBP}(\mathrm{mmHg})$ & $77.95 \pm 12.78$ & $76.29 \pm 12.37$ & 6.79 & $<0.001$ \\
\hline \multicolumn{5}{|l|}{ Blood lipid } \\
\hline Dyslipidemia (n/\%) & $7520 / 56.79$ & $7774 / 51.65$ & 74.76 & $<0.001$ \\
\hline Hypertriglyceridemia(n/\%) & $5730 / 43.27$ & $5272 / 35.03$ & 201.33 & $<0.001$ \\
\hline Hypercholesteremia (n/\%) & $3416 / 25.80$ & $3454 / 22.95$ & 31.04 & $<0.001$ \\
\hline High LDL-C(n/\%) & $2902 / 22.30$ & $3195 / 21.90$ & 0.64 & 0.42 \\
\hline Low HDL-C (n/\%) & $624 / 4.71$ & $587 / 3.90$ & 9.77 & 0.002 \\
\hline \multicolumn{5}{|l|}{ Distribution of FBG } \\
\hline$<6.1 \mathrm{mmol} / \mathrm{L}(\mathrm{n} / \%)$ & $11143 / / 84.15$ & $13154 / 87.41$ & 102.02 & $<0.001$ \\
\hline $6.1-6.9 \mathrm{mmol} / \mathrm{L}(\mathrm{n} / \%)$ & $1183 / 8.93$ & $1103 / 7.32$ & & \\
\hline$\geq 7.0 \mathrm{mmol} / \mathrm{L}(\mathrm{n} / \%)$ & $916 / 6.92$ & $793 / 5.27$ & & \\
\hline Uric acid（umol/L） & $356.36 \pm 94.50$ & $343.48 \pm 95.36$ & 11.39 & $<0.001$ \\
\hline $\begin{array}{l}\text { Abnormal liver function } \\
(\mathrm{n} / \%)\end{array}$ & $4899 / 37.11$ & $4525 / 30.21$ & 152.88 & $<0.001$ \\
\hline Albumin $(\mathrm{g} / \mathrm{L})$ & $45.91 \pm 2.73$ & $45.93 \pm 2.76$ & -0.58 & 0.56 \\
\hline Hemoglobin $(\mathrm{g} / \mathrm{L})$ & $146.86 \pm 15.61$ & $143.64 \pm 15.98$ & 16.82 & $<0.001$ \\
\hline $\begin{array}{l}\text { Abnormal renal } \\
\text { function }(\mathrm{n} / \%)\end{array}$ & $272 / 2.05$ & $264 / 1.75$ & 3.41 & 0.07 \\
\hline Fatty liver disease(n/\%) & $4940 / 37.31$ & $4704 / 31.26$ & 114.75 & $<0.001$ \\
\hline Gallbladder polyp(n/\%) & $1202 / 9.08$ & $1229 / 8.17$ & 7.45 & 0.006 \\
\hline Gallstone (n/\%) & $471 / 3.56$ & $546 / 3.63$ & 0.103 & 0.75 \\
\hline Renal calculus(n/\%) & $565 / 4.27$ & $570 / 3.79$ & 4.2 & 0.04 \\
\hline $\begin{array}{l}\text { Abnormalbone mineral } \\
\text { density }(\mathrm{n} / \%)\end{array}$ & $3578 / 75.71$ & $3582 / 74.44$ & 2.06 & 0.15 \\
\hline Osteopenia (n/\%) & $2287 / 48.39$ & $2301 / 47.82$ & 0.32 & 0.56 \\
\hline Osteoporosis (n/\%) & $1291 / 27.32$ & $1281 / 26.62$ & 0.59 & 0.44 \\
\hline Thyroid nodule (n/\%) & $3488 / 35.82$ & $4015 / 38.99$ & 21.47 & $<0.001$ \\
\hline
\end{tabular}




\begin{tabular}{lcccc} 
Lung lesions(n/\%) & $646 / 5.31$ & $665 / 4.92$ & 1.94 & 0.16 \\
Gastric lesions(n/\%) & $356 / 36.59$ & $337 / 35.03$ & 0.51 & 0.48 \\
$\begin{array}{l}\text { Intestinal lesions(n/\%) } \\
\text { abnormal cervical }\end{array}$ & $60 / 25.42$ & $49 / 23.22$ & 0.32 & 0.57 \\
$\begin{array}{l}\text { liquid-based cytological } \\
\text { test(n/\%) }\end{array}$ & $16 / 0.65$ & $35 / 0.90$ & 1.36 & 0.31 \\
$\begin{array}{l}\text { Breast lesions(n/\%) } \\
\text { abnormal gynecological }\end{array}$ & $1872 / 62.32$ & $2882 / 63.10$ & 0.48 & 0.49 \\
$\begin{array}{l}\text { ultrasonography(n/\%) } \\
\text { Prostatic lesion(n/\%) }\end{array}$ & $1463 / 50.71$ & $2299 / 52.20$ & 1.74 & 0.19 \\
\hline
\end{tabular}

Abbreviations:BMI,body mass index ;SBP,Systolic blood pressure;DBP,Diastolic blood pressure; LDL-C,low density lipoprotein cholesterol ;HDL-C,high density lipoprotein cholesterol ;FBG,fasting blood-glucose. 
Table 2.Regression analysis of food-specific IgG and various healthy outcomes

\begin{tabular}{|c|c|c|c|c|}
\hline Health outcomes & $\begin{array}{l}\text { Odds ratio } \\
(95 \% \mathrm{CI}))\end{array}$ & $\mathrm{p}$ value & $\begin{array}{c}\text { Adjusted odds ratio } \\
(95 \% \mathrm{CI}))\end{array}$ & $\mathrm{p}$ value \\
\hline Overweight & $0.81(0.78-0.85)$ & $<0.001$ & $0.92(0.87-0.96)$ & $<0.001$ \\
\hline Central obesity & $0.87(0.83-0.91)$ & $<0.001$ & $0.99(0.94-1.04)$ & 0.59 \\
\hline Dyslipidemia & $0.86(0.82-0.90)$ & $<0.001$ & $0.96(0.91-1.0)$ & 0.07 \\
\hline Hypercholesterolemia & $0.89(0.85-0.94)$ & $<0.001$ & $0.95(0.90-1.0)$ & 0.07 \\
\hline Hypertriglyceridemia & $0.79(0.75-0.83)$ & $<0.001$ & $0.87(0.83-0.92)$ & $<0.001$ \\
\hline Low HDL-C & $0.90(0.81-1.0)$ & 0.04 & $0.98(0.88-1.09)$ & 0.65 \\
\hline Pathoglycemia & $0.83(0.77-0.89)$ & $<0.001$ & $0.93(0.87-0.99)$ & 0.03 \\
\hline Hyperuricemia & $0.91(0.86-0.96)$ & 0.001 & $1.06(0.99-1.13)$ & 0.1 \\
\hline Dysarteriotony & $0.86(0.81-0.91)$ & $<0.001$ & $0.98(0.92-1.04)$ & 0.47 \\
\hline Abnormal SBP & $0.87(0.81-0.93)$ & $<0.001$ & $098(0.91-1.05)$ & 0.53 \\
\hline Abnormal DBP & $0.83(0.87-0.88)$ & $<0.001$ & $0.93(0.87-1.0)$ & 0.05 \\
\hline Anemia & $1.23(1.11-1.45)$ & 0.001 & $1.15(1.01-1.33)$ & 0.05 \\
\hline $\begin{array}{l}\text { Abnormal liver } \\
\text { function }\end{array}$ & $0.85(0.81-0.89)$ & $<0.001$ & $0.98(0.92-1.04)$ & 0.47 \\
\hline Fatty liver disease & $0.83(0.79-0.81)$ & $<0.001$ & $0.96(0.90-1.03)$ & 0.28 \\
\hline Gallbladder polyp & $0.98(0.91-1.06)$ & 0.58 & $1.02(0.95-1.01)$ & 0.56 \\
\hline Kidney stone & $0.95(0.86-1.06)$ & 0.34 & $1.02(0.91-1.14)$ & 0.69 \\
\hline Thyroid nodule & $1.10(1.05-1.16)$ & $<0.001$ & $1.09(1.04-1.15)$ & 0.001 \\
\hline Prostatic lesion & $0.96(0.88-1.04)$ & 0.34 & $1.01(0.93-1.11)$ & 0.78 \\
\hline
\end{tabular}

Abbreviations: CI, confidence interval; OR, odds ratio;HDL-C,high density lipoprotein cholesterol ;BP,blood pressure;SBP,Systolic blood pressure;

DBP,Diastolic blood pressure;FBG,fasting blood-glucose.Adjusted for age, sex(except sex-specific models), body mass index(except BMI-specific models), waistline(except waistline-specific models), blood pressure(except BP-specific models), blood lipid(except blood lipid specific models), FBG(except FBG-specific models).Fatty liver and uric acid were also introduced as covariates in the model with liver function and kidney stones as outcomes.

Complete covariate data available for 10637 person examinations. 

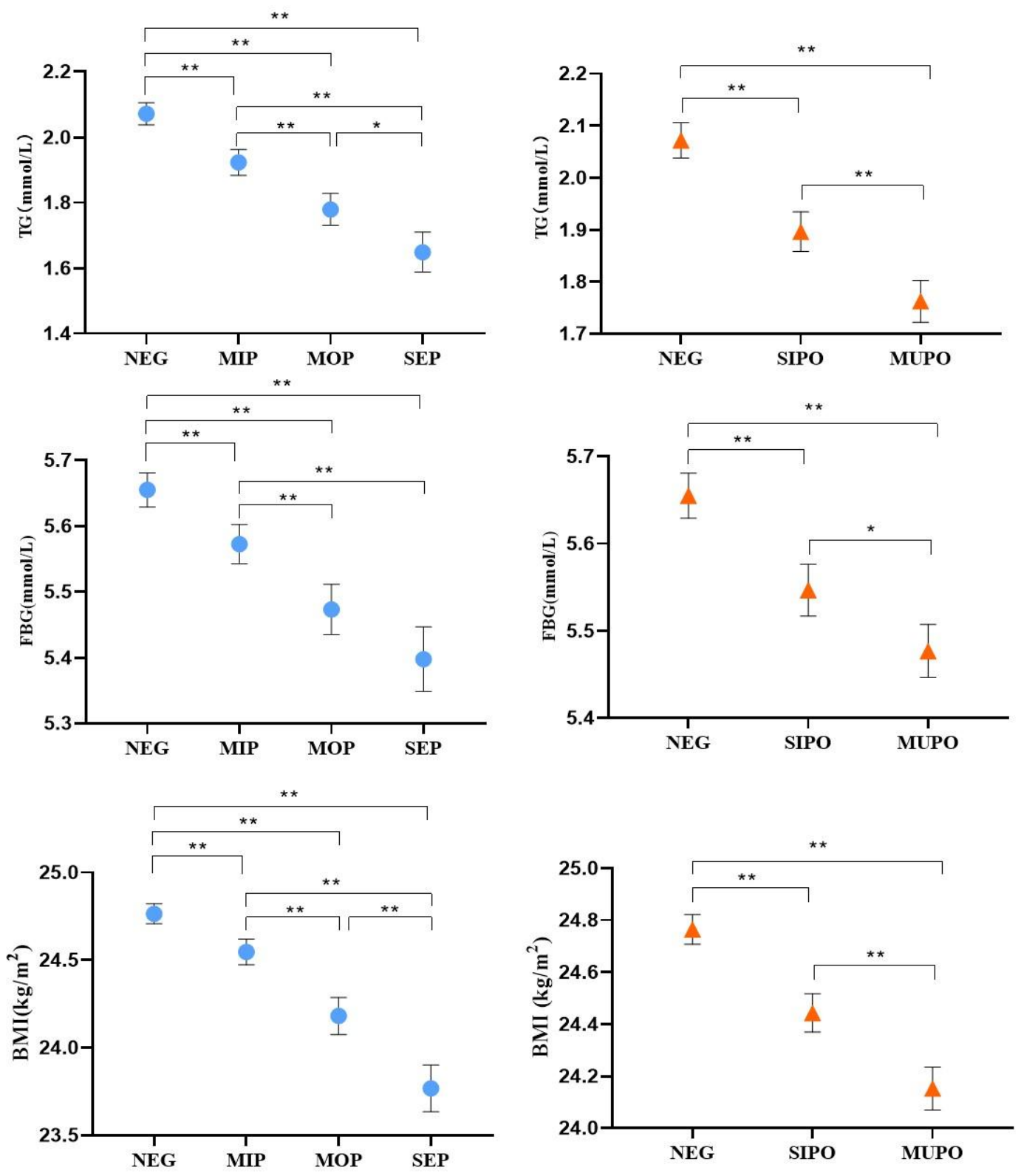

Figure 3.Relations of different degrees and types of food-specific IgG with FBG,TG and BMI.(Median and 95\%CI.NEG:Negative for food-specific IgG,MIP:Mild positive for food-specific IgG,MOP:Moderate positive for food-specific IgG,SEP:Severe positive for food-specific IgG,SIPO:A food-specific IgG positive;MUPO:Multiple food-specific IgG positive;BMI,body mass index;FBG,fasting blood-glucose;TG,triglyceride .* means $\mathrm{P}<0.05$ and $* *$ means $\mathrm{P}<0.001$.) 


\section{References}

1. Xiao N, Liu F, Zhou G, Sun M, Ai F, Liu Z. Food-specific IgGs Are Highly Increased in the Sera of Patients with Inflammatory Bowel Disease and Are Clinically Relevant to the Pathogenesis. Intern Med. 2018;57(19):2787-98.

2. Wang G, Ren J, Li G, Hu Q, Gu G, Ren H, et al. The utility of food antigen test in the diagnosis of Crohn's disease and remission maintenance after exclusive enteral nutrition. Clin Res Hepatol Gastroenterol. 2018;42(2):145-52.

3. Uzunismail H, Cengiz M, Uzun H, Ozbakir F, Goksel S, Demirdag F, et al. The effects of provocation by foods with raised IgG antibodies and additives on the course of Crohn's disease: a pilot study. Turk J Gastroenterol. 2012;23(1):19-27.

4. Cai C, Shen J, Zhao D, Qiao Y, Xu A, Jin S, et al. Serological investigation of food specific immunoglobulin $\mathrm{G}$ antibodies in patients with inflammatory bowel diseases. PLoS One. 2014;9(11):e112154.

5. Cappelletti M, Tognon E, Vona L, Basello K, Costanzi A, Speciani MC, et al. Food-specific serum IgG and symptom reduction with a personalized, unrestricted-calorie diet of six weeks in Irritable Bowel Syndrome (IBS). Nutr Metab (Lond). 2020;17(1):101.

6. Geiselman JF. The Clinical Use of IgG Food Sensitivity Testing with Migraine Headache Patients: a Literature Review. Curr Pain Headache Rep. 2019;23(11):79.

7. Finocchi C, Sivori G. Food as trigger and aggravating factor of migraine. Neurol Sci. 2012;33 Suppl 1:S77-80.

8. Tao R, Fu Z, Xiao L. Chronic Food Antigen-specific IgG-mediated Hypersensitivity Reaction as A Risk Factor for Adolescent Depressive Disorder. Genomics Proteomics Bioinformatics. 2019;17(2):183-89.

9. Karakula-Juchnowicz H, Galecka M, Rog J, Bartnicka A, Lukaszewicz Z, Krukow P, et al. The Food-Specific Serum IgG Reactivity in Major Depressive Disorder Patients, Irritable Bowel Syndrome Patients and Healthy Controls. Nutrients. 2018;10(5).

10. Severance EG, Dupont D, Dickerson FB, Stallings CR, Origoni AE, Krivogorsky $\mathrm{B}$, et al. Immune activation by casein dietary antigens in bipolar disorder. Bipolar Disord. 2010;12(8):834-42.

11. Karakula-Juchnowicz H, Szachta P, Opolska A, Morylowska-Topolska J, Galecka M, Juchnowicz D, et al. The role of IgG hypersensitivity in the pathogenesis and therapy of depressive disorders. Nutr Neurosci. 2017;20(2):110-18.

12. Bentz S, Hausmann M, Piberger H, Kellermeier S, Paul S, Held L, et al. Clinical relevance of IgG antibodies against food antigens in Crohn's disease: a double-blind cross-over diet intervention study. Digestion. 2010;81(4):252-64.

13. Atkinson W, Sheldon TA, Shaath N, Whorwell PJ. Food elimination based on IgG antibodies in irritable bowel syndrome: a randomised controlled trial. Gut. 2004;53(10):1459-64.

14. Mitchell N, Hewitt CE, Jayakody S, Islam M, Adamson J, Watt I, et al. Randomised controlled trial of food elimination diet based on IgG antibodies for the prevention of migraine like headaches. Nutr J. 2011;10:85. 
15. Xie Y, Zhou G, Xu Y, He B, Wang Y, Ma R, et al. Effects of Diet Based on IgG Elimination Combined with Probiotics on Migraine Plus Irritable Bowel Syndrome. Pain Res Manag. 2019;2019:7890461.

16. Jian L, Anqi H, Gang L, Litian W, Yanyan X, Mengdi W, et al. Food Exclusion Based on IgG Antibodies Alleviates Symptoms in Ulcerative Colitis: A Prospective Study. Inflamm Bowel Dis. 2018;24(9):1918-25.

17. Gunasekeera V, Mendall MA, Chan D, Kumar D. Treatment of Crohn's Disease with an IgG4-Guided Exclusion Diet: A Randomized Controlled Trial. Dig Dis Sci. 2016;61(4):1148-57.

18. Wilders-Truschnig M, Mangge H, Lieners C, Gruber H, Mayer C, Marz W. IgG antibodies against food antigens are correlated with inflammation and intima media thickness in obese juveniles. Exp Clin Endocrinol Diabetes. 2008;116(4):241-5.

19. Burton OT, Tamayo JM, Stranks AJ, Koleoglou KJ, Oettgen HC. Allergen-specific IgG antibody signaling through FcgammaRIIb promotes food tolerance. J Allergy Clin Immunol. 2018;141(1):189-201 e3.

20. Burton OT, Logsdon SL, Zhou JS, Medina-Tamayo J, Abdel-Gadir A, Noval Rivas M, et al. Oral immunotherapy induces $\mathrm{IgG}$ antibodies that act through FcgammaRIIb to suppress IgE-mediated hypersensitivity. J Allergy Clin Immunol. 2014;134(6):1310-17 e6.

21. Orgel K, Burk C, Smeekens J, Suber J, Hardy L, Guo R, et al. Blocking antibodies induced by peanut oral and sublingual immunotherapy suppress basophil activation and are associated with sustained unresponsiveness. Clin Exp Allergy. 2019;49(4):461-70.

22. Tsai M, Mukai K, Chinthrajah RS, Nadeau KC, Galli SJ. Sustained successful peanut oral immunotherapy associated with low basophil activation and peanut-specific IgE. J Allergy Clin Immunol. 2020;145(3):885-96 e6.

23. Wang HY, Li Y, Li JJ, Jiao CH, Zhao XJ, Li XT, et al. Serological investigation of IgG and IgE antibodies against food antigens in patients with inflammatory bowel disease. World J Clin Cases. 2019;7(16):2189-203.

24. Shakoor Z, AlFaifi A, AlAmro B, AlTawil LN, AlOhaly RY. Prevalence of IgG-mediated food intolerance among patients with allergic symptoms. Ann Saudi Med. 2016;36(6):386-90.

25. Coucke F. Food intolerance in patients with manifest autoimmunity. Observational study. Autoimmun Rev. 2018;17(11):1078-80.

26. Vojdani A. Molecular mimicry as a mechanism for food immune reactivities and autoimmunity. Altern Ther Health Med. 2015;21 Suppl 1:34-45.

27. Jansen A, Mandic AD, Bennek E, Frehn L, Verdier J, Tebrugge I, et al. Anti-food and anti-microbial IgG subclass antibodies in inflammatory bowel disease. Scand $\mathrm{J}$ Gastroenterol. 2016;51(12):1453-61.

28. Lin S, Yang X, Xing Y, Wang X, Li Y. The Clinical Application Value of Multiple Combination Food Intolerance Testing. Iran J Public Health. 2019;48(6):1068-73.

29. Batista NV, Pereira RV, Noviello ML, Dourado LP, Perez DA, Foureaux G, et al. Prolonged ingestion of ovalbumin diet by sensitized mice improves the metabolic consequences induced by experimental food allergy. Clin Exp Immunol. 
2014;178(3):416-27.

30. Munoz-Cano R, San Bartolome C, Casas-Saucedo R, Araujo G, Gelis S, Ruano-Zaragoza $\mathrm{M}$, et al. Immune-Mediated Mechanisms in Cofactor-Dependent Food Allergy and Anaphylaxis: Effect of Cofactors in Basophils and Mast Cells. Front Immunol. 2020;11:623071.

31. Sato S, Yanagida N, Ebisawa M. How to diagnose food allergy. Curr Opin Allergy Clin Immunol. 2018;18(3):214-21.

32. Rochelle P. Using randomised, double-blind, N-of-1 trials of food challenge to diagnose food allergy and assess the effectiveness of food allergen avoidance. J R Soc Med. 2018;111(1):31-32.

33. Kanagaratham C, El Ansari YS, Lewis OL, Oettgen HC. IgE and IgG Antibodies as Regulators of Mast Cell and Basophil Functions in Food Allergy. Front Immunol. 2020;11:603050.

34. Savilahti EM, Rantanen V, Lin JS, Karinen S, Saarinen KM, Goldis M, et al. Early recovery from cow's milk allergy is associated with decreasing $\operatorname{IgE}$ and increasing IgG4 binding to cow's milk epitopes. J Allergy Clin Immunol. 2010;125(6):1315-21 e9.

35. Ohsaki A, Venturelli N, Buccigrosso TM, Osganian SK, Lee J, Blumberg RS, et al. Maternal IgG immune complexes induce food allergen-specific tolerance in offspring. J Exp Med. 2018;215(1):91-113. 